ARTICLE

\title{
A previously unrecognized membrane protein in the Rhodobacter sphaeroides LH1-RC photocomplex
}

\author{
Kazutoshi Tani (1) ${ }^{1,8}$, Kenji V. P. Nagashima², Ryo Kanno ${ }^{3}$, Saki Kawamura ${ }^{4}$, Riku Kikuchi ${ }^{4}$, Malgorzata Hall ${ }^{3}$, \\ Long-Jiang Yu (D) ${ }^{5}$, Yukihiro Kimura (i) ${ }^{6}$, Michael T. Madigan ${ }^{7}$, Akira Mizoguchi ${ }^{1}$, Bruno M. Humbel ${ }^{3}$ \& \\ Zheng-Yu Wang-Otomo (1) 4,8凶
}

Rhodobacter ( $R b a$.) sphaeroides is the most widely used model organism in bacterial photosynthesis. The light-harvesting-reaction center (LH1-RC) core complex of this purple phototroph is characterized by the co-existence of monomeric and dimeric forms, the presence of the protein PufX, and approximately two carotenoids per LH1 $\alpha \beta$-polypeptides. Despite many efforts, structures of the Rba. sphaeroides LH1-RC have not been obtained at high resolutions. Here we report a cryo-EM structure of the monomeric LH1-RC from Rba. sphaeroides strain IL106 at $2.9 \AA$ resolution. The LH1 complex forms a C-shaped structure composed of $14 \alpha \beta$-polypeptides around the RC with a large ring opening. From the cryo-EM density map, a previously unrecognized integral membrane protein, referred to as protein-U, was identified. Protein- $U$ has a U-shaped conformation near the LH1-ring opening and was annotated as a hypothetical protein in the Rba. sphaeroides genome. Deletion of protein- $U$ resulted in a mutant strain that expressed a much-reduced amount of the dimeric LH1-RC, indicating an important role for protein- $U$ in dimerization of the LH1-RC complex. PufX was located opposite protein- $U$ on the LH1-ring opening, and both its position and conformation differed from that of previous reports of dimeric LH1-RC structures obtained at lowresolution. Twenty-six molecules of the carotenoid spheroidene arranged in two distinct configurations were resolved in the Rba. sphaeroides LH1 and were positioned within the complex to block its channels. Our findings offer an exciting new view of the core photocomplex of Rba. sphaeroides and the connections between structure and function in bacterial photocomplexes in general.

\footnotetext{
${ }^{1}$ Graduate School of Medicine, Mie University, Tsu 514-8507, Japan. ${ }^{2}$ Research Institute for Integrated Science, Kanagawa University, 2946 Tsuchiya, Hiratsuka, Kanagawa 259-1293, Japan. ${ }^{3}$ Imaging Section, Research Support Division, Okinawa Institute of Science and Technology Graduate University (OIST), 1919-1, Tancha, Onna-son, Kunigami-gun, Okinawa 904-0495, Japan. ${ }^{4}$ Faculty of Science, Ibaraki University, Mito 310-8512, Japan. ${ }^{5}$ Photosynthesis Research Center, Key Laboratory of Photobiology, Institute of Botany, Chinese Academy of Sciences, Beijing 100093, China. ${ }^{6}$ Department of Agrobioscience, Graduate School of Agriculture, Kobe University, Nada, Kobe 657-8501, Japan. ${ }^{7}$ School of Biological Sciences, Department of Microbiology, Southern Illinois University, Carbondale, IL 62901, USA. ${ }^{8}$ These authors jointly supervised: Kazutoshi Tani, Zheng-Yu Wang-Otomo. ${ }^{凶}$ email: ktani@doc.medic.mie-u.ac.jp; wang@ml.ibaraki.ac.jp
} 
$R$ hodobacter (Rba.) sphaeroides is a purple phototrophic bacterium widely distributed in natural habitats. This organism is extraordinarily versatile in its abilities to grow by any of five metabolic modes: photoautotrophically $\left(\mathrm{CO}_{2} /\right.$ light/ anoxic); photoheterotrophically (organic compounds/light/ anoxic); chemolithotrophically $\left(\mathrm{CO}_{2}+\mathrm{H}_{2} /\right.$ dark/oxic); and by the respiration (dark/oxic) or fermentation (dark/anoxic) of organic compounds $^{1}$. Rba. sphaeroides has been the most thoroughly investigated purple phototroph and has been widely used as a model for fundamental biochemical genetic studies of photochemistry, metabolism, and regulation. A crystal structure of the reaction center (RC) complex from Rba. sphaeroides was the second such to appear ${ }^{2,3}$ following shortly after that of the RC from Blastochloris (Blc.) viridis ${ }^{4}$. The two structures displayed high similarities in the arrangements of most proteins and cofactors, suggesting that the core components of the RC-the Lsubunit, M-subunit and cofactors-were structurally conserved among all purple bacteria ${ }^{5}$.

The core light-harvesting (LH1) system of Rba. sphaeroides is unusual. The LH1 complex composed of $\alpha$ - and $\beta$-polypeptides forms an S-shaped structure in the native membranes ${ }^{6,7}$ and $2 \mathrm{D}$ crystals $^{8-10}$. The S-shaped LH1 incorporates two RCs resulting in a so-called dimeric LH1-RC complex, a structure that was also observed in Rba. blasticus ${ }^{11}$ and Rhodobaca bogoriensis ${ }^{12}$, and was suggested to exist in a few other purple bacteria ${ }^{13}$. A key component thought to be responsible for the dimerization is PufX, a protein present within the LH1-RC core complex ${ }^{14,15}$. PufX is an 82-amino-acid polypeptide encoded by the pufX gene in the Rba. sphaeroides photosynthetic gene cluster ${ }^{16}$. PufX is present in all species of Rhodobacter ${ }^{17}$ and has been shown to be essential for phototrophic growth in both Rba. sphaeroides and Rba. capsulatus ${ }^{18-20}$. Previous work has probed the structural and functional role of PufX and implicated this key protein in regulation of membrane morphology, core photocomplex organization, and cyclic electron transfer ${ }^{21}$.

In addition to the dimeric core complex, a monomeric LH1-RCPufX complex also exists in native membranes of Rba. sphaeroides and Rba. blasticus and can be separately purified from the dimeric form $8,11,13$. The monomeric core complex is identical to one-half of a dimer with a gap in the LH1 ring, displaying a C-shaped arrangement ${ }^{11}$. It was demonstrated that monomers can reform dimers upon reconstitution, suggesting that the monomer represents a native state in equilibrium with the dimeric complexes ${ }^{8}$. Indeed, only the monomeric form has been observed for the core complexes from Rba. veldkampii ${ }^{22-24}$ and Rba. capsulatus ${ }^{13}$. In addition to its structure, Rba. sphaeroides LH1 is also unique in its carotenoid amount. Regardless of dimeric or monomeric form, the Rba. sphaeroides LH1 complex contains approximately two carotenoids per $\alpha \beta$-polypeptides ${ }^{25-28}$, the highest ratio among known LH1 complexes of purple phototrophs. However, despite the likely importance of carotenoids in LH1 function, Rba. sphaeroides LH1 carotenoids have not been resolved from low-resolution structural analyses (an 8 - $\AA$ structure is the best thus far attained) ${ }^{27}$; thus, the precise position and function of carotenoids in the Rba. sphaeroides LH1 remains unknown.

Here we present a robust cryo-EM structure of the monomeric core complex from Rba. sphaeroides f. sp. denitrificans (strain IL106) ${ }^{29}$. A previously unrecognized integral membrane protein with a U-shaped conformation (designated as protein- $U$ ) was discovered in the LH1-RC structure and located to one side of the LH1 opening. Protein- $U$ had been annotated as a hypothetical protein in the Rba. sphaeroides genome and is encoded in all Rba. sphaeroides strains with published genome sequences, implying a structural and functional role for this protein in the core complex. Our cryo-EM structure also reveals a precise conformation of PufX that definitively locates this crucial protein to a different position in the complex than previously proposed. Finally, our structure allowed all carotenoids in the LH1 to be resolved and positioned in unique arrangements. Our findings shed new light on the key photosynthetic component of an old organism and provide a major new view of structure-function relationships in the core photocomplexes of photosynthetic bacteria.

\section{Results}

Structural overview. The cryo-EM structure of the monomeric LH1-RC complex of Rba. sphaeroides IL106 was determined at $2.9 \AA$ resolution (Fig. 1, Supplementary Table 1 and Supplementary Figs. 1-4). The LH1 complex is composed of 14 pairs of helical $\alpha \beta$-polypeptides, 28 BChls $a$ and 26 spheroidenes surrounding the $\mathrm{RC}$ with a large opening in the slightly elliptical C-shaped ring structure. Although structures of the LH1 $\alpha \beta$ polypeptides and all proteins in the $\mathrm{RC}$ are consistent with previous work, it is notable that PufX was found at a different position and with a different conformation from that in the dimeric structure (PDB: 4JC9, 4JCB) determined at $8 \AA$ for the Rba. sphaeroides LH1-RC (strain DBC $\Omega$ G, Supplementary Fig. 5a) ${ }^{27}$. In addition, a large fragment of electron potential densities near the LH1-ring opening was observed in the refined map but could not be modeled by any known proteins or cofactors. Because our density map at $2.9 \AA$ resolution was of very high quality, we were able to trace not only the main chain of this protein but also the sidechains for many residues. The derived amino acid sequence allowed us to search the Rba. sphaeroides IL106 genome (GenBank assembly accession: GCA_003363065.1), and in doing so, a hypothetical protein (DWF04_22265) containing 53 amino acids emerged whose sequence turned out to perfectly fit the density map (Fig. 2b). Hereafter, we refer to this previously unrecognized protein as "protein-U", the seventh distinct protein in the Rba. sphaeroides LH1-RC complex (Fig. 1c, d; Fig. 2a, b).

The Rba. sphaeroides LH1 a $\beta$-polypeptides form a roughly elliptical C-shaped arrangement with long and short dimensions of $122 \AA$ and $116 \AA$ (Fig. 1b), respectively, for the outer $\beta$ polypeptide ring (distances measured between the outer edges of opposite helices). The LH1 a-polypeptides are formylated at their $\mathrm{N}$-terminus as confirmed by TOF/MS (Supplementary Fig. 6) and the cryo-EM density map (Supplementary Fig. 4). These formyl groups were modeled in the LH1 structure and play a role in stabilizing the $\mathrm{N}$-terminal regions of the a-polypeptides through hydrogen bonding. PufX (chain X) and protein-U (chain $\mathrm{U}$ ) are located opposite each other on the two sides of the LH1-ring opening (Fig. 1, 2a). As a result, the positions of several LH1 $\alpha \beta$ subunits near protein-U gradually deviated from the closed LH1 ellipse (Fig. 1d) that has been observed in the monomeric type LH1-RCs from Thermochromatium (Tch.) tepidum ${ }^{30}$ and Rhodopseudomonas (Rps.) palustris ${ }^{31}$. Molecules of BChl $a$ in the Rba. sphaeroides LH1 are ligated by His residues with average His- $\mathrm{Mg}$ distances of $2.6 \AA$ ( $\alpha$-His32) and $2.2 \AA$ ( $\beta$-His38), and have average $\mathrm{Mg}-\mathrm{Mg}$ distances of $9.6 \AA$ within a dimer and $8.4 \AA$ between dimers (Fig. 1c, Supplementary Table 2). Cofactors in the RC include four BChls $a$, two bacteriopheophytins (BPhe) $a$, and one 15-cis-spheroidene and ubiquinone (UQ)-10 molecules at both the $\mathrm{Q}_{\mathrm{A}}$ and $\mathrm{Q}_{\mathrm{B}}$ site. Non-heme iron and the head group of UQ in the RC are aligned along a line parallel to that connecting PufX and protein-U. A total of three UQ-10, four cardiolipins, and 13 phosphatidylglycerols were modeled in the LH1-RC structure of Rba. sphaeroides IL106. The numbers of phospholipids are in agreement with those determined from biochemical analysis $^{32}$, and one of the cardiolipins was found at the same position as that reported previously in an RC-only structure from Rba. sphaeroides ${ }^{33}$. 

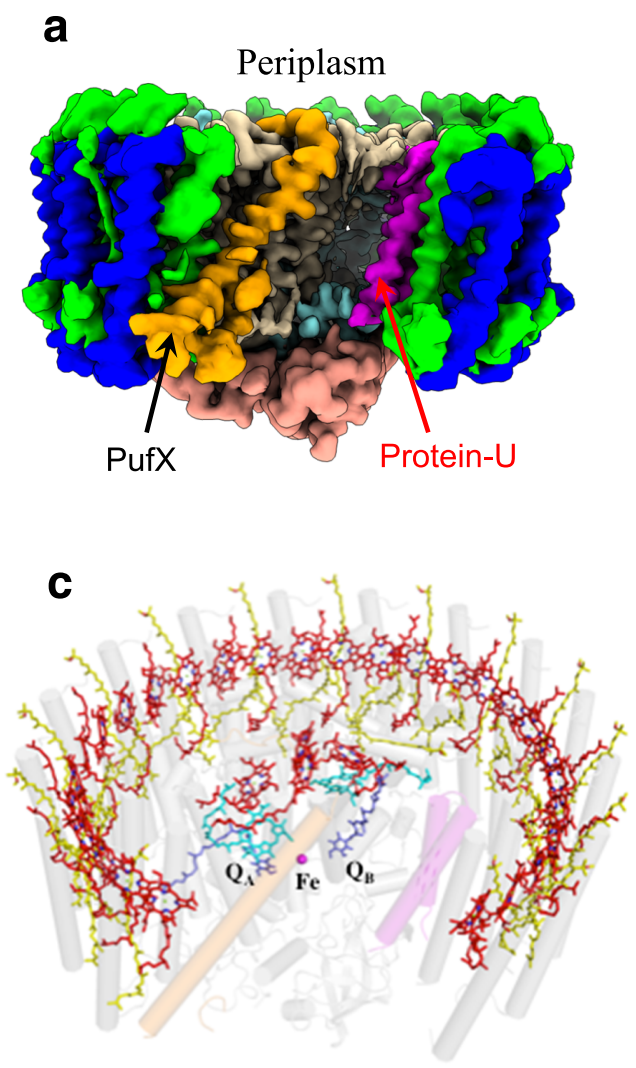
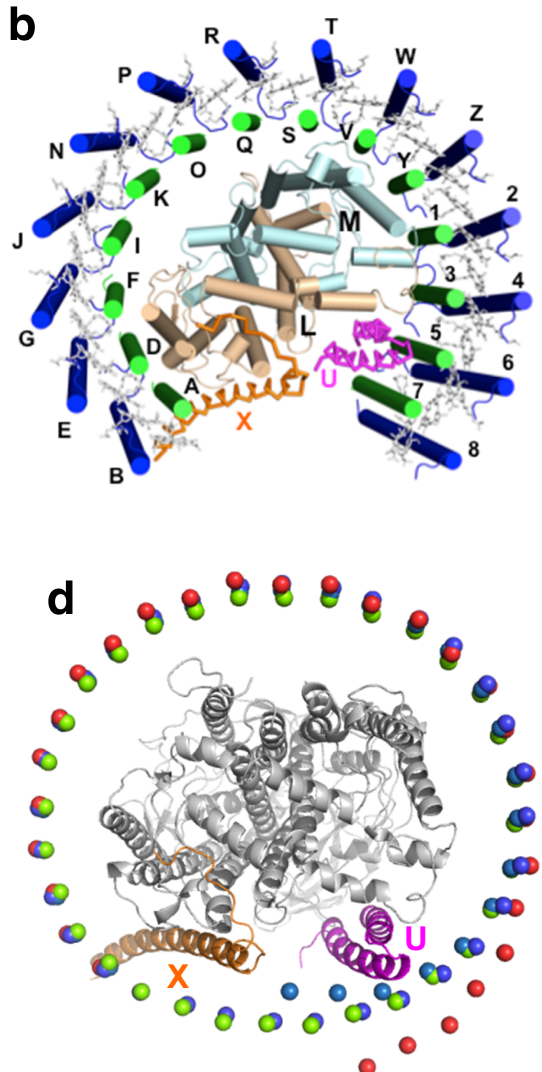

Fig. 1 Structure overview of the monomeric LH1-RC complex from Rba. sphaeroides IL106. a Side view of surface representations for the LH1-RC proteins parallel to the membrane plane. $\mathbf{b}$ Top view of the LH1-RC proteins from the periplasmic side of the membrane. Labels indicate chain IDs, and the RC $\mathrm{H}$-subunit was omitted for clarity. Color scheme: LH1- $\alpha$, green; LH1- $\beta$, blue; RC-L, wheat; RC-M, cyan; RC-H, salmon; PufX, orange; protein-U, magenta. c Tilted view of the cofactor arrangement viewing from the periplasmic side. Color scheme: $\mathrm{BChl} a$, red sticks; spheroidenes, yellow sticks; BPhe $a$, cyan sticks; UQ-10, blue sticks; PufX, transparent orange cylinder; protein-U, transparent magenta cylinder, and transparent gray cylinders for all other proteins. d Comparisons of the central $\mathrm{Mg}$ atom positions in the LH1 BChl a molecules from Rba. sphaeroides IL106 (red spheres) with those from Tch. tepidum (green spheres, PDB: 5Y5S), protein-W-containing Rps. palustris (cyan spheres, PDB: 6Z5S), and protein-W-deficient Rps. palustris (blue spheres, PDB: 6Z5R). The structures were superimposed by $\mathrm{C} \alpha$ carbons of the $R C(L, M, H)$ proteins. The ribbon models represent the RC-L, $-M$ and $-H$ subunits (gray), PufX (orange), and protein-U (magenta) of the Rba. sphaeroides IL106.

The unidentified protein-U. Rba. sphaeroides protein- $\mathrm{U}$ is located in the interior of the LH1-RC complex between the RCL subunit and two LH1 a-polypeptides and exhibits a helixturn-helix conformation with both $\mathrm{N}$ - and C-termini on the cytoplasmic side (Fig. 2a, b). The two transmembrane (TM) helical regions contain highly hydrophobic residues rich in Gly and Ala, and are connected by a short loop of five residues. The characteristic U-shaped feature was clearly observed in the cryo-EM map and is consistent with that predicted by the membrane protein topology program TMHMM $^{34}$ (Fig. 2d). Protein- $U$ interacts with surrounding proteins mainly through its terminal domains and loop region. The main chain oxygen of protein-U Val4 and the sidechain carboxyl group of Glu6 form hydrogen bonds with the sidechain of Arg15 in an $\alpha$ polypeptide, while a segment (Thr49-Pro50-Asn51) in the protein-U C-terminal region forms hydrogen bonds with two a-polypeptides (chains 5 and 7) (Fig. 1b, 2c). In the loop region of protein-U, Trp32 and Phe33 form close contacts with Trp265 and Trp266 in the RC-L subunit where a putative UQ-10 molecule is present nearby. The unique position and interaction pattern of protein- $U$ indicate that one of its functions may be as a "spacer" pushing several nearby LH1 polypeptides outward from the RC as shown in Fig. 1d and implying a structural role in producing steric hindrances to prevent the formation of a closed LH1 ring.
To further investigate potential functions of protein- $U$, we constructed a mutant strain (IL106- $\Delta \mathrm{U}$ ) deficient in protein-U (Supplementary Fig. 7). This deletion mutant was capable of phototrophic growth at a growth rate similar to that of wild-type cells. However, levels of dimeric LH1-RC significantly decreased in the mutant compared with the wild type. Sucrose density gradient analysis revealed that the ratio of dimeric to monomeric LH1-RC was cut by nearly two-thirds ( 0.44 to 0.15$)$ in the mutant (Fig. 2e, Supplementary Fig. 8a), indicating that protein-U likely plays structural and functional roles in the stabilization of the monomer and formation of the dimers of the LH1-RC complex. No apparent effects of the absence of protein- $U$ on absorption spectra of either dimeric or monomeric LH1-RCs were detected (Supplementary Fig. 8b).

The PufX polypeptide. The full-length PufX of Rba. sphaeroides IL106 is encoded by 82 amino acids in which the N-terminal Met and C-terminal 13 residues are post-translationally removed in the expressed polypeptide as confirmed by TOF/MS (Fig. 3d, Supplementary Fig. 6). The PufX sequence of strain IL106 differs slightly from that of the type strain 2.4.1 at Asp58 and Pro61, where both are replaced with Gln in Rba. sphaeroides strain 2.4.1. In our cryo-EM structure, the N-terminal 15 residues of the expressed PufX were invisible presumably due to disordered conformations. PufX is located on the opposite side of protein- $\mathrm{U}$ 
a

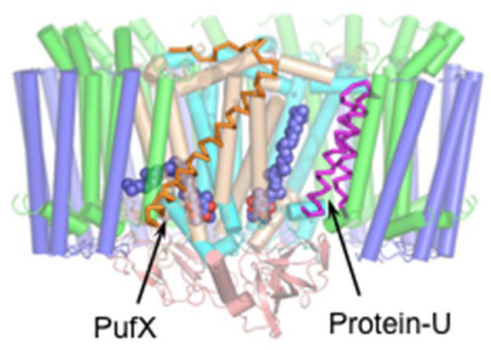

b

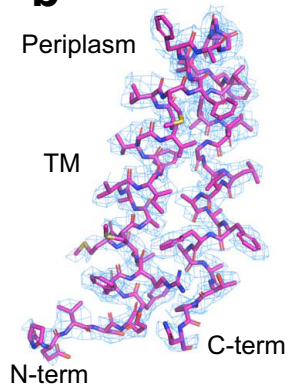

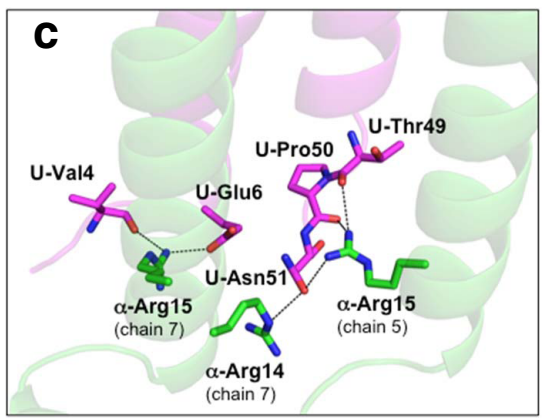

e

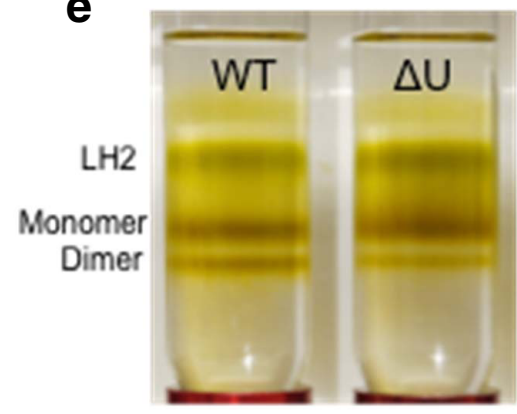

Fig. 2 Structure and property of $\boldsymbol{R}$ ba. sphaeroides protein-U. a Relative position of protein- $U$ (magenta ribbon) in the LH1-RC. PufX is shown by the orange ribbon and the $U Q-10$ at $Q_{A}$ and $Q_{B}$ sites are shown by blue spheres. All other proteins are shown by cylinders with the same color scheme as in Fig. 1(a). b Structure of protein- $U$ and its density map at a contour level of $3.0 \sigma$. c Close contacts ( $<3.5 \AA$, dashed lines) between the protein- $U$ terminal regions (magenta) and $\mathrm{LH} 1 \alpha$-polypeptides (green). d Amino acid sequence of protein- $\mathrm{U}$ and its two consecutive transmembrane regions predicted by the membrane protein topology program TMHMM. e Sucrose density gradient (10-40\% w/v) centrifugations of the solubilized pigment-protein complexes from wild-type (WT) and protein-U-deleted $(\Delta U)$ Rba. sphaeroides IL106 membranes.

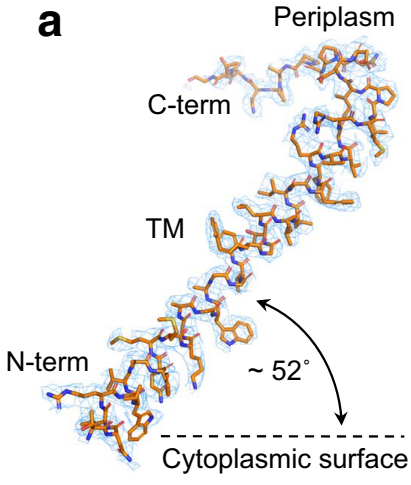

d d

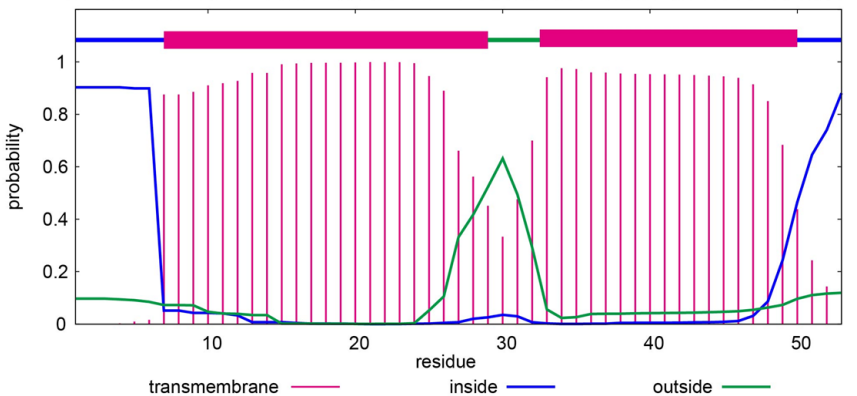

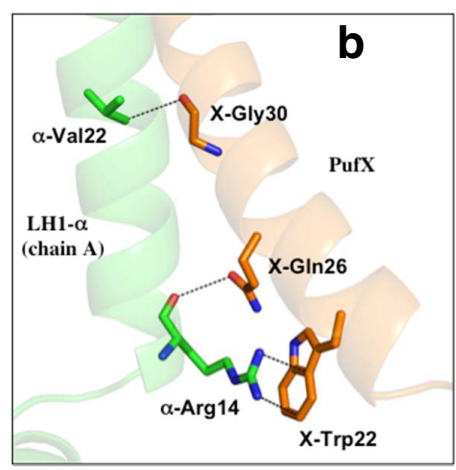

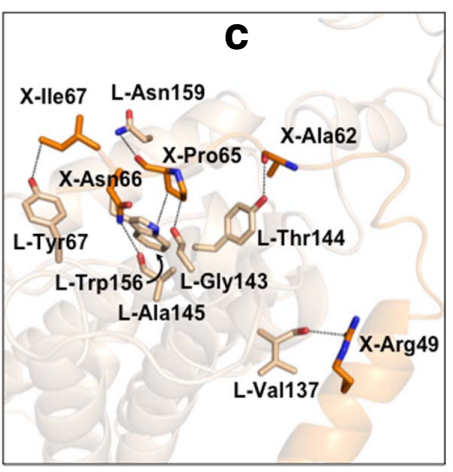

60

70 80 MADKTIFNDHLNTNPKTNLRLWVAFQMMKGAGWAGGVFFGTLLLIGFFRVVGRMLPIDENPAPAPNITGALETGIELIKHLV

Fig. 3 Structure and interactions of Rba. sphaeroides PufX. a Structure and density map (3.0 $\sigma$ ) of PufX with orientation toward the presumed membrane plane. b Close contacts ( $<3.5 \AA$, dashed lines) between the PufX N-terminal region (transparent orange) and an LH1 $\alpha$-polypeptide (chain ID: A, transparent green). c Close contacts ( $<3.5 \AA$, dashed lines) between the PufX C-terminal domain (transparent orange) and the RC L-subunit (transparent wheat). d Primary sequence of the full-length PufX in Rba. sphaeroides IL106. Sequence for the expressed PufX is shown by larger bold fonts. Membranespanning residues are underlined. The Glu58 and Pro61 (blue fonts) are both replaced by Gln in the type strain 2.4.1.

in the LH1-ring opening (Fig. 2a) at the same position with almost the same conformation as that of another PufX-containing LH1-RC recently reported for Rba. veldkampii (Supplementary Fig. 5b) ${ }^{24}$. PufX has a single, unusually long transmembrane domain with its $\mathrm{N}$-terminus on the cytoplasmic side and C-terminus on the periplasmic surface (Fig. 3a). Differing from other proteins in the LH1-RC, PufX displays a remarkably tilted conformation for its transmembrane helical domain with an angle of approximately $52^{\circ}$ inclined toward the presumed membrane plane (Fig. 3a); this results in a much longer membrane-spanning region (38 residues, Supplementary Fig. 10c) in good agreement with an experimental estimation for the core segment in PufX ${ }^{35}$.

No direct interactions $(<4.0 \AA)$ were observed between PufX and pigment molecules (BChl $a$ and carotenoids). However, the $\mathrm{N}$-terminal region of PufX interacts with an LH1 a-polypeptide (chain A), while the C-terminal domain has contacts with the RC L-subunit on the periplasmic surface. The Trp22 in PufX likely has cation $-\pi$ interactions with the sidechain of Arg14 in an 

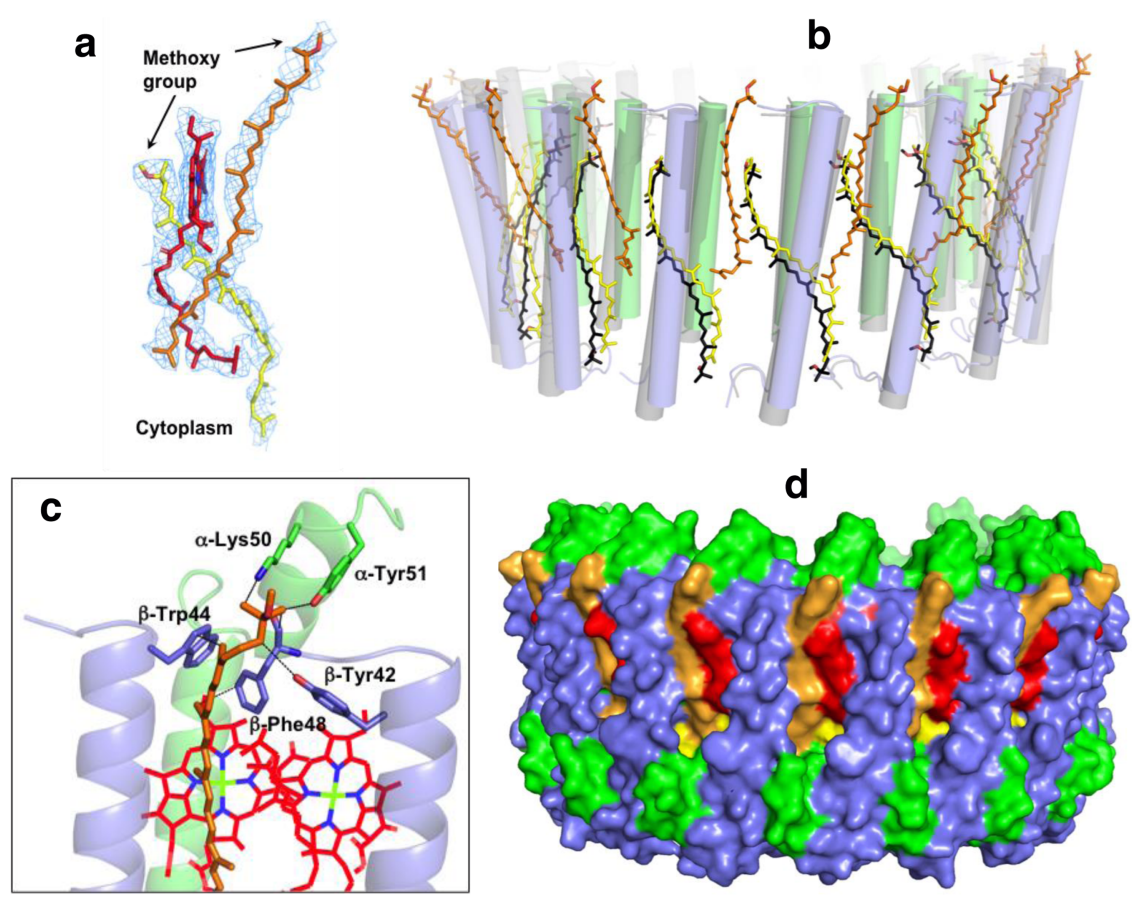

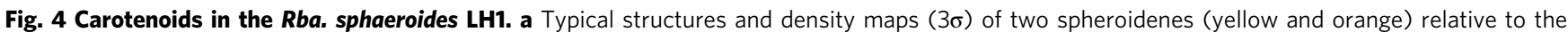
position of a BChl $a$ (red) in the LH1 complex with different configurations. $\mathbf{b}$ Two groups of the spheroidenes (yellow and orange sticks for Group-A and Group-B, respectively) in the Rba. sphaeroides LH1 in comparison with the carotenoids in the Tch. tepidum LH1 (black sticks, PDB:5Y5S). The structures were superimposed by $\mathrm{C} \alpha$ carbons of the LH1 $\alpha \beta$-polypeptides between Rba. sphaeroides (transparent green and slate-blue cylinders) and Tch. tepidum (transparent gray cylinders). c Close contacts (<4.0 $\AA$, dashed lines) between a Group-B spheroidene and its surrounding $\alpha \beta$-polypeptides. $\mathbf{d}$ Side view of the surface representation shows a sealed fence for the Rba. sphaeroides LH1 complex. Color scheme: LH1- $\alpha$, green; LH1- $\beta$, slate-blue; BChl $a$, red; spheroidenes, yellow (Group-A) and orange (Group-B).

a-polypeptide (Fig. 3b). The Arg49 and Ala62 in PufX form hydrogen bonds with Val137 and Thr144 in the RC L-subunit, respectively (Fig. 3c). The extensive interactions between the PufX C-terminal end (Ala62-Ile67) and a segment of the RC L-subunit (Gly143-Trp156) explain the experimental observation that the C-terminus of PufX plays an important role in dimerization and assembly of the LH1-RC in Rba. sphaeroides ${ }^{36}$.

Carotenoids in the Rba. sphaeroides LH1 Complex. A total of 26 all-trans-spheroidenes in the LH1 complex of Rba. sphaeroides IL106 were clearly resolved in our cryo-EM map (Fig. 1c, 4a, Supplementary Fig. 4). This corresponds to a ratio of approximately two carotenoids per $\alpha \beta$-polypeptides in contrast to the one carotenoid per $\alpha \beta$-pair commonly found in other LH1 complexes $24,30,31,37-39$. All methoxy groups of the Rba. sphaeroides carotenoids point toward the periplasmic side, and the carotenoids can be grouped into two sets based on their orientations and positions in the LH1. Group-A spheroidenes are embedded deeply in the transmembrane region between the $\alpha$ and $\beta$-polypeptides at similar positions and with a similar conformation to those in the LH1 complexes of other purple bacteria $^{24,30,31,37-40}$. A representative comparison is shown in Fig. $4 \mathrm{~b}$ for the conformations between the carotenoids in Rba. sphaeroides IL106 and that of Tch. tepidum. The methoxy groups of these carotenoids are positioned close to the center of bacteriochlorins (Fig. 4a) and there are no apparent associations between the carotenoids and surrounding polypeptides and BChl a molecules.

In contrast to Group-A spheroidenes, Group-B spheroidenes adopt a distinct configuration within the Rba. sphaeroides LH1 with a large shift toward the periplasmic side (Fig. $4 \mathrm{~b}$ ). Each of these carotenoids is located in between two adjacent $\alpha \beta$-pairs with their methoxy group protruded on the periplasmic surface. The methoxy ends of these carotenoids are in close proximity to the C-terminal ends of $\alpha \beta$-polypeptides (Fig. 4c) and the central portions form hydrophobic interactions with $\mathrm{BChl} a$ molecules. The Group-B spheroidenes, combined with Group-A spheroidenes, fill the space between adjacent $\alpha \beta$-subunits (Fig. 4d), and this leads to blockage of the pores (channels) observed in BChl $a$ containing LH1 complexes containing only Group-A carotenoids $30,31,38,39$. The combination of both Group A and B carotenoids results in a tightly sealed, impenetrable LH1 "fence" that requires alternative strategies for facilitating quinone transport between the Rba. sphaeroides RC and quinone pools during photosynthetic electron flow. These alternative strategies include the C-shaped monomeric LH1 whose structure was revealed herein or an S-shaped dimeric LH1 with incomplete rings as previously reported ${ }^{27}$.

\section{Discussion}

The gene encoding the 53 amino acids in protein- $\mathrm{U}$ is flanked in the Rba. sphaeroides IL106 genome by genes encoding a transglutaminase family protein and metallo-hydrolase and is the only gene in this region transcribed in the opposite direction (Supplementary Fig. 7). This suggests that transcription of the gene encoding protein- $U$ is controlled independently from that of adjacent genes. The expressed protein- $U$ locates in the interior space between LH1 (chains 5 and 7) and RC near the edge of the LH1-ring opening. The corresponding position is occupied by a cardiolipin molecule in the protein W-containing Rps. palustris LH1-RC that also has an opening in the LH1 ring ${ }^{31}$. Protein-U has a tandem arrangement of two membrane-spanning segments connected by a short loop, forming a U-shaped conformation. High frequencies of Gly and Ala residues in the transmembrane 
region indicate a tendency for these a helices to pack tightly at particular sites such as Gly22, Gly35, and Gly39 (Fig. 2d) to form a canonical left-handed supercoil as is often seen in $\alpha$-bundle membrane proteins ${ }^{41-43}$. Such helical pairs with antiparallel arrangements are known to form large interfacial areas, which in the case of protein-U may contribute to LH1-RC stabilization and/or facilitate dimerization of the complex.

A genomic database search of the genus Rhodobacter revealed that six of fifteen species contain protein- $U$ (or protein-U-like) genes, and all Rba. sphaeroides strains with sequenced genomes encode this protein (Supplementary Fig. 9a). Proteins-U can be classified into three types based on their sequences (Supplementary Fig. 9b): Type-1 (WP_002721225) for Rba. johrii, Rba. megalophilus and all but one Rba. sphaeroides strains; Type-2 (WP_176504535) for Rba. ovatus; and Type-3 (WP_085996593) for Rba. azotoformans, Rba. sediminicola and Rba. sphaeroides ATCC 17025. Type-1 and Type- 3 proteins-U have the same number of residues and share high sequence identity, whereas Type-2 has a long N-terminal domain but also shares high sequence identity with Type- 1 and Type-3 proteins- $U$ in other portions (Supplementary Fig. 9b). All Rhodobacter species containing protein- $U$ also have pufX (or pufX-like) genes but the converse is not true. Considering that a Rba. sphaeroides protein$\mathrm{U}$-deletion strain in our work and wild-type Rba. blasticus that naturally lacks protein- $\mathrm{U}^{11}$ are both capable of photosynthetic growth and producing at least some dimeric LH1-RC, protein- $\mathrm{U}$ is clearly not indispensable for photosynthesis as is true of the more widely distributed PufX; a PufX-deletion strain of $R b a$. sphaeroides (ATCC 17023) was reported to be photosynthetically incompetent ${ }^{19,44}$. Therefore, we conclude that major functions of protein-U should include (i) stabilization of the partially opened LH1-RC structures and (ii) enhancement of efficiency for dimerization of the LH1-RC complex from that formed spontaneously, suggesting that the dimeric form may be the most efficient (or preferred) state of the complex in Rba. sphaeroides.

The small size and extremely high hydrophobicity of $R b a$. sphaeroides protein-U likely hindered its early discovery by biochemical and structural analyses. The presence of an additional protein in dimers of the Rba. sphaeroides LH1-RC was previously suspected from extra residual densities in an 8.5 - $\AA$ cryo-EM projection map of $2 \mathrm{D}$ crystals from Rba. sphaeroides strain DD13/ $\mathrm{DGa} 2^{10}$. Two significant density features were identified within each half of the LH1-RC dimer, corresponding approximately to the positions of protein- $\mathrm{U}$ and PufX in our cryo-EM structure of the monomeric LH1-RC. One of the densities was tentatively proposed to be PufX ${ }^{10}$ but this corresponds to protein- $\mathrm{U}$ in our structure, while another relatively diffuse density feature was left unassigned $^{10}$ and this corresponds to PufX in our study. Subsequently, a 3D structure of the dimeric LH1-RC from Rba. sphaeroides strain DBC $\Omega \mathrm{G}$ was determined at $8 \AA$ using combined techniques ${ }^{27}$. However, PufX was found at yet a different position in this structure from those previously reported ${ }^{10}$ and in our work (Supplementary Fig. 5a). The two Rba. sphaeroides strains used in these studies both lacked a peripheral lightharvesting complex (LH2) but were mutant derivatives of the same parental strain (wild-type: NCIB8253) ${ }^{45}$. Thus, the conflicting results likely indicate the difficulty in obtaining a definitive structure of such a large photocomplex at limiting resolutions, although structural rearrangements induced by genetic manipulations and/or dimerization cannot be absolutely ruled out.

Structures for individual polypeptides of LH1- $\beta$ and PufX isolated from Rba. sphaeroides have been investigated using solution NMR. The $\beta$-polypeptide shows a single transmembrane a-helix starting from Leu19 with a remarkably high degree of disorder for the N-terminal domain in organic solvents ${ }^{46}$. The flexible and unstructured features for the N-terminal region of the $\beta$-polypeptides were also observed in our cryo-EM structure in which the first 5-10 residues were invisible in the density map. Subsequently, the solution structures of the $\beta$-polypeptide were further determined in organic and detergent solvents, both exhibiting two helical domains separated by a flexible region with largely bent conformations ${ }^{47,48}$ in contrast to that observed in our cryo-EM structure. Solution structures of PufX were also determined in organic solvents, yielding two quite different conformations: one showed two hydrophobic helices connected by a helical bend (PDB: 2ITA, 2NRG; Supplementary Fig. 10b) 49,50 , whereas a second revealed a rather straight, single continuous helix for the central transmembrane domain (PDB: 2DW3 $)^{51}$. The latter closely matches that in our cryo-EM structure over the a-helical region (Supplementary Fig. 10a).

Alignments of eighteen PufX and PufX-like sequences in the database revealed a well-conserved central helical domain flanked by flexible termini (Supplementary Fig. 10c), and the diversities in structure, function and interactions of PufX have been thoroughly reviewed ${ }^{21}$. Genetic studies have demonstrated that Rba. sphaeroides PufX is most tightly associated with the LH1-RC complex, less tightly bound to the LH1-only complex, and least tightly associated with RC-only preparations ${ }^{52}$. PufX tends to copurify with the LH1 a-polypeptide ${ }^{53}$, implying preferential interactions with the LH1 inner ring (chain A), consistent with our cryo-EM structure. Cross-species studies of PufX led to a hypothesis that in all Rhodobacter species PufX defines the composition of the monomeric LH1-RC complex, managing the aggregation state of the pigment proteins around the $\mathrm{RC}$ and determining the position of the LH1-ring opening relative to the entrance of the $\mathrm{Q}_{\mathrm{B}}$ pocket in the $\mathrm{RC}^{13}$. The nearly symmetric arrangements of PufX and protein- $U$ relative to the LH1-ring opening and the fact that deletion of protein- $U$ resulted in a greatly reduced amount of dimeric LH1-RC may suggest a similar and/or cooperative role for protein- $U$ in controlling the topology of the LH1 complex.

PufX in Rba. sphaeroides likely correlates with the carotenoid content in LH1. It was demonstrated that an engineered Rba. sphaeroides LH1 with a point-mutation (LH1-a Trp8 $\rightarrow$ Phe) produced fewer carotenoids and allowed photosynthetic growth in the absence of PufX ${ }^{54}$. This result was interpreted by assuming that an LH1 with fewer carotenoids would possess multiple pores (channels) in the closed complex as seen in the LH1 of Tch. tepidum $^{30}$ and that this would allow quinone transport through the pores in the absence of PufX and support photosynthetic growth. By contrast, a corresponding Rba. sphaeroides strain with a higher carotenoid content in its LH1 was photosynthetically incompetent ${ }^{54}$. This in turn suggested that the high carotenoid content (approximately two carotenoids per $\alpha \beta$-pair) in the native Rba. sphaeroides LH1 could block the LH1 channels (as confirmed in our structure, Fig. 4d) requiring alternative pathways for quinone transport in order to grow photosynthetically. Consequently, a PufX-containing C-shaped (monomeric) or S-shaped (dimeric) LH1 complex could fulfill this physiological requirement.

As experimental model systems, phototrophic purple bacteria such as Rba. sphaeroides continue to reveal the most intimate secrets of photosynthetic energy conversion, and in this regard, our work provides the most robust and detailed structure of the Rba. sphaeroides LH1-RC structure to date. Our cryo-EM structure not only identified a previously unsuspected integral membrane protein and characterized its likely functions but also determined the precise position and conformation of $\mathrm{PufX}-\mathrm{a}$ long-standing issue in bacterial photosynthesis-and resolved all carotenoids in the core complex. Our findings lay the foundation for an even deeper understanding of photosynthesis, in particular 
those aspects surrounding structure-function relationships in light-energy conversion, quinone transport, and regulation of carotenoid biosynthesis.

\section{Methods}

Preparation and characterization of the native LH1-RC complex. The Rba. sphaeroides f. sp. denitrificans (strain IL106) cells were cultivated phototrophically (anoxic/light) at room temperature for 7 days under incandescent light $(60 \mathrm{~W})$. This strain can also grow chemoheterotrophically using nitrate and dimethyl sulfoxide (DMSO) as the terminal electron acceptors (dark/anoxic) ${ }^{29}$. Preparation of the native LH1-RC was conducted by solubilizing chromatophores $\left(\mathrm{OD}_{870}\right.$ $\mathrm{nm}=40)$ with $1.0 \% \mathrm{w} / \mathrm{v} n$-dodecyl- $\beta$-D-maltopyranoside (DDM) in $20 \mathrm{mM}$ Tris$\mathrm{HCl}(\mathrm{pH} 8.0)$ buffer for $60 \mathrm{~min}$ at room temperature, followed by differential centrifugation. The supernatant was loaded onto a DEAE column (Toyopearl $650 \mathrm{~S}$, TOSOH) equilibrated at $4{ }^{\circ} \mathrm{C}$ with $20 \mathrm{mM}$ Tris- $\mathrm{HCl}$ buffer ( $\mathrm{pH} \mathrm{8.0)} \mathrm{con-}$ taining $0.1 \% \mathrm{w} / \mathrm{v}$ DDM. The fractions were eluted in an order of $\mathrm{LH} 2$, monomeric LH1-RC, and dimeric LH1-RC by a linear gradient of $\mathrm{NaCl}$ from $0 \mathrm{mM}$ to $400 \mathrm{mM}$. The peak fractions of monomeric LH1-RC were collected and further purified by sucrose gradient density centrifugation with five-stepwise sucrose concentrations $(10,17.5,25,32.5$, and $40 \% \mathrm{w} / \mathrm{v})$ in $20 \mathrm{mM}$ Tris-HCl buffer ( $\mathrm{pH} 8.0)$ containing $0.05 \% \mathrm{w} / \mathrm{v} \mathrm{DDM}$ at $4{ }^{\circ} \mathrm{C}$ and $150,000 \times g$ for $6 \mathrm{~h}$. The monomeric LH1-RC fractions were concentrated for absorption measurement and assessed by negative-stain EM using a JEM-1011 instrument (JEOL) (Supplementary Fig. 1a). We were unable to distinguish the native monomeric LH1-RC originally present in the intact membranes from those degraded from dimers during isolation and purification. Masses and composition of the LH1 and PufX polypeptides were measured by matrixassisted laser desorption/ionization time-of-flight mass spectroscopy (MALDITOF/MS) and reverse-phase HPLC (Supplementary Fig. 6b, 6c), respectively ${ }^{55}$ Quinones were extracted from chromatophores, purified LH1-RC, and RC-only complexes, and quinone contents were analyzed (Supplementary Fig. $6 \mathrm{~d}$ ) $^{56}$. Approximately six UQ-10 molecules were estimated per purified LH1-RC.

Mutagenesis and characterization. A $0.8-\mathrm{kb}$ DNA region at the direct upstream and a $0.9-\mathrm{kb}$ DNA region at the direct downstream of the protein- $U$ gene were amplified by PCR using Rba. sphaeroides IL106 genomic DNA as the template and the PCR primers shown in Supplementary Table 3. These DNA fragments were connected in a manner that excludes the protein- $U$ gene and cloned in a suicide vector $\mathrm{pJPCm}^{57}$ using an In-Fusion HD Cloning Kit (TAKARA BIO, Shiga, Japan) as shown in Supplementary Fig. 7. This plasmid was named pJPCm_ $\Delta U$, and then a DNA fragment containing $s a c R B$ genes (lethal genes under the presence of sucrose) and a kanamycin-resistant cartridge were inserted at the unique SacI restriction site on this plasmid. This plasmid was named pJPCm_ $\Delta \mathrm{U}-\mathrm{SKm}$ and introduced from the E. coli S17-1 $\lambda$ pir host cells by conjugational transfer into the cells of Rba. sphaeroides IL106 wild-type strain with a spontaneous resistance to rifampicin. The gene encoding protein- $\mathrm{U}$ was deleted from the Rba. sphaeroides genome via a two-step homologous recombination, which was screened by kanamycin resistance in the first step and by sucrose resistance in the second, as previously described ${ }^{57}$. After the conjugation $E$. coli cells were eliminated by addition of $50 \mu \mathrm{g} / \mathrm{ml}$ of rifampicin to the growth medium. The removal of the protein- $U$ gene without any insertions of antibiotics-resistant cartridges was confirmed by PCR and DNA sequencing experiments. This mutant was named IL106$\Delta \mathrm{U}$.

Cells of Rba. sphaeroides IL106- $\Delta \mathrm{U}$ were cultivated phototrophically at room temperature under the same condition as that for the wild-type. The chromatophores of both wild-type and IL106- $\Delta \mathrm{U}$ were treated with $1.0 \% \mathrm{w} / \mathrm{v}$ DDM in $20 \mathrm{mM}$ Tris- $\mathrm{HCl}$ ( $\mathrm{pH} 8.0$ ) buffer for $60 \mathrm{~min}$ at room temperature, followed by differential centrifugation. The supernatants $(1 \mathrm{~mL})$ with adjusted concentrations of $\mathrm{OD}_{870-\mathrm{nm}}=5,9,14$ were loaded on a stepwise sucrose gradient $(10,17.5,25,32.5$, and $40 \% \mathrm{w} / \mathrm{v})$ in $20 \mathrm{mM}$ Tris- $\mathrm{HCl}$ buffer $(\mathrm{pH} 8.0)$ containing $0.05 \% \mathrm{w} / \mathrm{v}$ DDM. Centrifugation was conducted at $4{ }^{\circ} \mathrm{C}$ and $150,000 \times \mathrm{g}$ for $6 \mathrm{~h}$. All of the dimeric and monomeric LH1-RC layers were carefully collected and a ratio of the dimeric to monomeric LH1-RC was calculated using their volumes and absorption intensities (Supplementary Fig. 8).

Cryo-EM data collection. Proteins for cryo-EM were concentrated to $\sim 3 \mathrm{mg} / \mathrm{ml}$ Two microliters of the protein solution were applied on glow-discharged holey carbon grids (200 mesh Quantifoil R2/2 molybdenum), which had been treated with $\mathrm{H}_{2}$ and $\mathrm{O}_{2}$ mixtures in a Solarus plasma cleaner (Gatan, Pleasanton, USA) for $30 \mathrm{~s}$ and then blotted, and plunged into liquid ethane at $-182{ }^{\circ} \mathrm{C}$ using an EM GP2 plunger (Leica, Microsystems, Vienna, Austria). The applied parameters were a blotting time of $6 \mathrm{~s}$ at $80 \%$ humidity and $4{ }^{\circ} \mathrm{C}$. Data were collected on a Talos Arctica (Thermo Fisher Scientific, Hillsboro, USA) electron microscope at $200 \mathrm{kV}$ equipped with a Falcon 3 camera (Thermo Fisher Scientific) (Supplementary Fig. 1). Movies were recorded using EPU software (Thermo Fisher Scientific) at a nominal magnification of $92 \mathrm{k}$ in counting mode and a pixel size of $1.094 \AA$ at the specimen level with a dose rate of 0.98 e- per physical pixel per second, corresponding to 0.82 e- per $\AA^{2}$ per second at the specimen level. The exposure time was $51 \mathrm{~s}$, resulting in an accumulated dose of 42 e- per $\AA^{2}$. Each movie includes 40 fractioned frames.
Image processing. All of the stacked frames were subjected to motion correction with MotionCor2 ${ }^{58}$. Defocus was estimated using CTFFIND4 ${ }^{59}$. A total of 551,846 particles were selected from 2,766 micrographs using the EMAN2 suite (Supplementary Fig. 2) ${ }^{60}$. The initial 3-D model was generated with 36,284 particles from 87 selected micrographs with underfocus values ranging between 2 and $3 \mu \mathrm{m}$ using EMAN2. All of the picked particles were further analyzed with RELION3.0 and $3.1^{61}$, and 219,927 particles were selected by $2 \mathrm{D}$ classification and divided into four classes by $3 \mathrm{D}$ classification resulting in only one good class containing 160,488 particles. The $3 \mathrm{D}$ auto refinement without any imposed symmetry $(\mathrm{C} 1)$ produced a map at $3.10 \AA$ resolution after contrast transfer function refinement, Bayesian polishing, masking, and post-processing. The selected 160,448 particle projections were subjected to subtraction of the detergent micelle density followed by 3D auto refinement to yield the final map with a resolution of $2.94 \AA$ according to the goldstandard Fourier shell correlation using a criterion of 0.143 (Supplementary Fig. 2) ${ }^{62}$. The local resolution maps were calculated on RESMAP63.

Model building and refinement of the LH1-RC complex. The atomic models of the LH1 ring of the Tch. tepidum LH1-RC (PDB: 5Y5S) and the RC of the Rba. sphaeroides (PDB: 1PCR) was fitted to the cryo-EM map obtained for the Rba. sphaeroides LH1-RC using Chimera ${ }^{64}$. Amino acid substitutions and real-space refinement for the peptides and cofactors were performed using COOT ${ }^{65}$. Whole regions of PufX and protein- $\mathrm{U}$ as well as both terminal regions of the LH1 $\alpha$ subunit were modeled ab-initio based on the density. The manually modified model was refined in real-space on PHENIX ${ }^{66}$, and the COOT/PHENIX refinement was iterated until the refinements converged. Finally, the statistics calculated using MolProbity ${ }^{67}$ were checked. Figures were drawn with the Pymol Molecular Graphic System (Schrödinger) ${ }^{68}$ and UCSF Chimera ${ }^{64}$.

Reporting Summary. Further information on research design is available in the Nature Research Reporting Summary linked to this article.

\section{Data availability}

Map and model have been deposited in the EMDB and PDB with the accession codes: EMD-31400 and 7F0L. The coordinates (5Y5S) for Tch. tepidum LH1-RC is available at PDB. All other data are available from the corresponding authors upon reasonable request.

Received: 15 June 2021; Accepted: 1 October 2021; Published online: 02 November 2021

\section{References}

1. Mackenzie, C. et al. The home stretch, a first analysis of the nearly completed genome of Rhodobacter sphaeroides 2.4.1. Photosynth. Res. 70, 19-41 (2001).

2. Allen, J. P., Feher, G., Yeates, T. O., Komiya, H. \& Rees, D. C. Structure of the reaction center from Rhodobacter sphaeroides R-26: The cofactors. Proc. Natl Acad. Sci. USA. 84, 5730-5734 (1987).

3. Allen, J. P., Feher, G., Yeates, T. O., Komiya, H. \& Rees, D. C. Structure of the reaction center from Rhodobacter sphaeroides R-26: The protein subunits. Proc. Natl Acad. Sci. USA. 84, 6162-6166 (1987).

4. Deisenhofer, J., Epp, O., Miki, K., Huber, R. \& Michel, H. Structure of the protein subunits in the photosynthetic reaction centre of Rhodopseudomonas viridis at $3 \AA$ resolution. Nature 318, 618-624 (1985).

5. Olson, T. L., Williams, J. C. \& Allen, J. F. The three-dimensional structures of bacterial reaction centers. Photosynth. Res. 120, 87-98 (2014).

6. Jungas, C., Ranck, J.-L., Rigaud, J.-L., Joliot, P. \& Vermeglio, A. Supramolecular organization of the photosynthetic apparatus of Rhodobacter sphaeroides. EMBO J. 18, 534-542 (1999).

7. Bahatyrova, S. et al. The native architecture of a photosynthetic membrane. Nature 430, 1058-1062 (2004).

8. Scheuring, S. et al. Structural role of PufX in the dimerization of the photosynthetic core complex of Rhodobacter sphaeroides. J. Biol. Chem. 279, 3620-3626 (2004).

9. Siebert, C. A. et al. Molecular architecture of photosynthetic membranes in Rhodobacter sphaeroides: the role of PufX. EMBO J. 23, 690-700 (2004).

10. Qian, P., Hunter, C. N. \& Bullough, P. A. The 8.5 A projection structure of the core RC-LH1-PufX dimer of Rhodobacter sphaeroides. J. Mol. Biol. 349, 948-960 (2005).

11. Scheuring, S., Busselez, J. \& Levy, D. Structure of the dimeric PufX-containing core complex of Rhodobacter blasticus by in situ atomic force microscopy. J. Biol. Chem. 280, 1426-1431 (2005).

12. Semchonok, D. A., Chauvin, J.-P., Frese, R. N., Jungas, C. \& Boekema, E. J. Structure of the dimeric RC-LH1-PufX complex from Rhodobaca bogoriensis investigated by electron microscopy. Philos. Trans. R. Soc. B 367, 3412-3419 (2012). 
13. Crouch, L. I. \& Jones, M. R. Cross-species investigation of the function of the Rhodobacter PufX polypeptide and the composition of the RC-LH1 core complex. Biochim. Biophys. Acta - Bioenerg. 1817, 336-352 (2012).

14. Francia, F. et al. The reaction center-LH1 antenna complex of Rhodobacter sphaeroides contains one PufX molecule which is involved in dimerization of this complex. Biochemistry 38, 6834-6845 (1999).

15. Francia, F., Wang, J., Zischka, H., Venturoli, G. \& Oesterhelt, D. Role of the $\mathrm{N}$ - and C-terminal regions of the PufX protein in the structural organization of the photosynthetic core complex of Rhodobacter sphaeroides. Eur. J. Biochem. 269, 1877-1885 (2002).

16. Lee, J. K., DeHoff, B. S., Donohue, T. J., Gumport, R. I. \& Kaplan, S. Transcriptional analysis of puf operon expression in Rhodobacter sphaeroides 2. 4.1 and an intercistronic transcription terminator mutant. J. Biol. Chem. 264, 19354-19365 (1989).

17. Tsukatani, Y. et al. Phylogenetic distribution of unusual triheme to tetraheme cytochrome subunit in the reaction center complex of purple bacteria. Photosynth. Res. 79, 83-91 (2004).

18. Klug, G. \& Cohen, S. N. Pleiotropic effects of localized Rhodobacter capsulatus puf operon deletions on production of light-absorbing pigment-protein complexes. J. Bacteriol. 170, 5814-5821 (1988).

19. Farchaus, J. W., Barz, W. P., Grünberg, H. \& Oesterhelt, D. Studies on the PufX polypeptide and its requirement for photoheterotrophic growth in Rhodobacter sphaeroides. EMBO J. 11, 2779-2788 (1992).

20. Lilburn, T. G., Haith, C. E., Prince, R. C. \& Beatty, J. T. Pleiotropic effects of pufX gene deletion on the structure and function of the photosynthetic apparatus of Rhodobacter capsulatus. Biochim. Biophys. Acta - Bioenerg. 1100, 160-170 (1992).

21. Holden-Dye, K., Crouch, L. I. \& Jones, M. R. Structure, function and interaction of the PufX protein. Biochim. Biophys. Acta - Bioenerg. 1777, 613-630 (2008).

22. Busselez, J. et al. Structural basis for the PufX-mediated dimerization of bacterial photosynthetic core complex. Structure 15, 1674-1683 (2007).

23. Liu, L.-N., Sturgis, J. N. \& Scheuring, S. Native architecture of the photosynthetic membrane from Rhodobacter veldkampii. J. Struct. Biol. 173, 138-145 (2011).

24. Bracun, L. et al. Cryo-EM structure of the photosynthetic RC-LH1-PufX supercomplex at 2.8-Å resolution. Sci. Adv. 7, eabf8864 (2021).

25. Sistrom, W. R. Control of antenna pigment components. in The Photosynthetic Bacteria (eds. Clayton, R. K. \& Sistrom, W. R.) 841-848 (Plenum Press, New York, 1978).

26. Broglie, R. M. et al. Isolation and characterization of the pigment-protein complexes of Rhodopseudomonas sphaeroides by lithium dodecyl sulfate/ polyacrylamide gel electrophoresis. Proc. Natl Acad. Sci. USA 77, 87-91 (1980).

27. Qian, P. et al. Three-dimensional structure of the Rhodobacter sphaeroides RC-LH1-PufX complex: Dimerization and quinone channels promoted by PufX. Biochemistry 52, 7575-7585 (2013).

28. Nagashima, K. V. P. et al. Probing structure-function relationships in early events in photosynthesis using a chimeric photocomplex. Proc. Natl Acad. Sci. U. S. A. 114, 10906-10911 (2017).

29. Satoh, T., Hoshino, Y. \& Kitamura, H. Rhodopseudomonas sphaeroides forma sp. denitrificans, a denitrifying strain as a subspecies of Rhodopseudomonas sphaeroides. Arch. Microbiol. 108, 265-269 (1976).

30. Yu, L.-J., Suga, M., Wang-Otomo, Z.-Y. \& Shen, J.-R. Structure of photosynthetic LH1-RC supercomplex at $1.9 \AA$ resolution. Nature 556, 209-213 (2018).

31. Swainsbury, D. J. K. et al. Structures of Rhodopseudomonas palustris RC-LH1 complexes with open or closed quinone channels. Sci. Adv. 7, eabe2631 (2021).

32. Nagatsuma, S. et al. Phospholipid distributions in purple phototrophic bacteria and LH1-RC core complexes. Biochim. Biophys. Acta - Bioenerg. 1860, 461-468 (2019).

33. McAuley, K. E. et al. Structural details of an interaction between cardiolipin and an integral membrane protein. Proc. Natl Acad. Sci. U. S. A. 96, 14706-14711 (1999).

34. Krogh, A., Larsson, B., von Heijne, G. \& Sonnhammer, E. L. L. Predicting transmembrane protein topology with a hidden Markov model: application to complete genomes. J. Mol. Biol. 305, 567-580 (2001)

35. Parkes-Loach, P. S. et al. Role of the core region of the PufX protein in inhibition of reconstitution of the core light-harvesting complexes of Rhodobacter sphaeroides and Rhodobacter capsulatus. Biochemistry 40, 5593-5601 (2001).

36. Qian, P., Martin, E. C., Ng, I. W. \& Hunter, C. N. The C-terminus of PufX plays a key role in dimerisation and assembly of the reaction center lightharvesting 1 complex from Rhodobacter sphaeroides. Biochim. Biophys. Acta Bioenerg. 1858, 705-803 (2017).

37. Qian, P., Siebert, C. A., Wang, P., Canniffe, D. P. \& Hunter, C. N. Cryo-EM structure of the Blastochloris viridis LH1-RC complex at $2.9 \AA$ A Nature 556, 203-208 (2018).
38. Tani, K. et al. Cryo-EM structure of a $\mathrm{Ca}^{2+}$-bound photosynthetic LH1-RC complex containing multiple $\alpha \beta$-polypeptides. Nat. Commun. 11, 4955 (2020).

39. Tani, K. et al. Cryo-EM structure of the photosynthetic LH1-RC complex from Rhodospirillum rubrum. Biochemistry. 60, 2483-2491 (2021).

40. Xin, Y. et al. Cryo-EM structure of the RC-LH core complex from an early branching photosynthetic prokaryote. Nat. Commun. 9, 1568 (2018).

41. Javadpour, M. M., Eilers, M., Groesbeek, M. \& Smith, S. O. Helix packing in polytopic membrane proteins: role of glycine in transmembrane helix association. Biophys. J. 77, 1609-1618 (1999).

42. Eilers, M., Patel, A. B., Liu, W. \& Smith, S. O. Comparison of helix interactions in membrane and soluble a-bundle proteins. Biophys. J. 82, 2720-2736 (2002).

43. Dong, H., Sharma, M., Zhou, H.-X. \& Cross, T. A. Glycines: role in $\alpha$-helical membrane protein structures and a potential indicator of native conformation. Biochemistry 51, 4779-4789 (2012).

44. Farchaus, J. W., Gruenberg, H. \& Oesterhelt, D. Complementation of a reaction center-deficient Rhodobacter sphaeroides pufLMX deletion strain in trans with pufBALM does not restore the photosynthesis-positive phenotype J. Bacteriol. 172, 977-985 (1990).

45. Jones, M. R. et al. Mutants of Rhodobacter sphaeroides lacking one or more pigment-protein complexes and complementation with reaction centre, LH1, and LH2 genes. Mol. Microbiol. 6, 1173-1184 (1992).

46. Kikuchi, J. et al. A light-harvesting antenna protein retains its folded conformation in the absence of protein-lipid and protein-pigment interactions. Biopolymers 49, 361-372 (1999).

47. Conroy, M. J. et al. The solution structure of Rhodobacter sphaeroides LH1ß reveals two helical domains separated by a more flexible region: structural consequences for the LH1 complex. J. Mol. Biol. 298, 83-94 (2000).

48. Sorgen, P. L. et al. Structure of the Rhodobacter sphaeroides light-harvesting 1 $\beta$ subunit in detergent micelles. Biochemistry 41, 31-41 (2002).

49. Tunnicliffe, R. B., Ratcliffe, E. C., Hunter, C. N. \& Williamson, M. P. The solution structure of the PufX polypeptide from Rhodobacter sphaeroides. FEBS Lett. 580, 6967-6971 (2006).

50. Ratcliffe, E. C. et al. Experimental evidence that the membrane-spanning helix of PufX adopts a bent conformation that facilitate dimerisation of the Rhodobacter sphaeroides RC-LH1 complex through N-terminal interactions. Biochim. Biophys. Acta - Bioenerg. 1807, 95-107 (2011).

51. Wang, Z.-Y., Suzuki, H., Kobayashi, M. \& Nozawa, T. Solution structure of the Rhodobacter sphaeroides PufX membrane protein: Implications for the quinone exchange and protein-protein interactions. Biochemistry 46, 3635-3642 (2007)

52. Pugh, R. J., McGlynn, P., Jones, M. R. \& Hunter, C. N. The LH1-RC core complex of Rhodobacter sphaeroides: interaction between components, timedependent assembly, and topology of the PufX protein. Biochim. Biophys. Acta - Bioenerg. 1366, 301-316 (1998).

53. Recchia, P. A. et al. Isolation of the PufX protein from Rhodobacter capsulatus and Rhodobacter sphaeroides: Evidence for its interaction with the apolypeptide of the core light-harvesting complex. Biochemistry 37, 11055-11063 (1998).

54. Olsen, J. D., Martin, E. C. \& Hunter, C. N. The PufX quinone channel enables the light-harvesting 1 antenna to bind more carotenoids for light collection and photoprotection. FEBS Lett. 591, 573-580 (2017).

55. Sekine, F. et al. Gene sequencing and characterization of the light-harvesting complex 2 from thermophilic purple sulfur bacterium Thermochromatium tepidum. Photosynth. Res. 111, 9-18 (2012).

56. Kimura, Y. et al. Characterization of the quinones in purple sulfur bacterium Thermochromatium tepidum. FEBS Lett. 589, 1761-1765 (2015).

57. Ohmine, M. et al. Cytochrome $c_{4}$ can be involved in the photosynthetic electron transfer system in the purple bacterium Rubrivivax gelatinosus. Biochemistry 48, 9132-9139 (2009).

58. Zheng, S. Q. et al. MotionCor2: anisotropic correction of beam-induced motion for improved cryo-electron microscopy. Nat. Methods 14, 331-332 (2017).

59. Rohou, A. \& Grigorieff, N. Fast and accurate defocus estimation from electron micrographs. J. Struct. Biol. 192, 216-221 (2015).

60. Tang, G. et al. EMAN2: an extensible image processing suite for electron microscopy. J. Struct. Biol. 157, 38-46 (2007).

61. Zivanov, J. et al. New tools for automated high-resolution cryo-EM structure determination in RELION-3. eLife 7, e42166 (2018).

62. Rosenthal, P. B. \& Henderson, R. Optimal determination of particle orientation, absolute hand, and contrast loss in single-particle electron cryomicroscopy. J. Mol. Biol. 333, 721-745 (2003).

63. Kucukelbir, A., Sigworth, F. J. \& D., T. H. Quantifying the local resolution of cryo-EM density maps. Nat. Methods 11, 63-65 (2014).

64. Pettersen, E. F. et al. UCSF Chimera-a visualization system for exploratory research and analysis. J. Comput. Chem. 25, 1605-1612 (2004).

65. Emsley, P., Lohkamp, B., Scott, W. G. \& Cowtan, K. Features and development of Coot. Acta Cryst. D66, 486-501 (2010).

66. Adams, P. D. et al. PHENIX: a comprehensive Python-based system for macromolecular structure solution. Acta Cryst. D66, 213-221 (2010). 
67. Chen, V. B. et al. MolProbity: all-atom structure validation for macromolecular crystallography. Acta Crystallogr. D. Biol. Crystallogr. 66 12-21 (2010).

68. DeLano, W. L. The PyMOL molecular graphics system (DeLano Scientific, LCC, San Carlos, 2004).

\section{Acknowledgements}

We thank Megumi Kobayashi, Ayumi Imai, and Risa Onuma for providing excellent technical assistances. This research was partially supported by Platform Project for Supporting Drug Discovery and Life Science Research (Basis for Supporting Innovative Drug Discovery and Life Science Research (BINDS)) from AMED under Grant Numbers JP20am0101118 (support number 1758) and JP20am0101116 (support number 1878), 17am0101116j0001, 18am0101116j0002, and 19am0101116j0003. R.K., M.H., and B.M.H. acknowledge the generous support of the Okinawa Institute of Science and Technology and the Japanese Cabinet Office. L.-J.Y. acknowledges support of the National Key R\&D Program of China (No. 2019YFA0904600). This work was supported in part by JSPS KAKENHI Grant Numbers JP16H04174, JP18H05153, JP20H05086, and JP20H02856, Takeda Science Foundation, and the Kurata Memorial Hitachi Science and Technology Foundation, Japan.

\section{Author contributions}

Z.-Y.W.-O. and K.T. designed the work, K.T., K.V.P.N., R. K., S.K., R. K., and M.H. performed the experiments, K.T., K.V.P.N., R. K., L.-J.Y., Y.K., M.T.M., A.M., B.M.H and Z.-Y.W.-O. analyzed data, Z.-Y.W.-O., K.T., K.V.P.N., and M.T.M. wrote the manuscript. All authors edited and revised the manuscript.

\section{Competing interests}

The authors declare no competing interests.

\section{Additional information}

Supplementary information The online version contains supplementary material available at https://doi.org/10.1038/s41467-021-26561-9.

Correspondence and requests for materials should be addressed to Kazutoshi Tani or Zheng-Yu Wang-Otomo.

Peer review information Nature Communications thanks Dmitry Semchonok and the other, anonymous, reviewer(s) for their contribution to the peer review of this work. Peer reviewer reports are available.

Reprints and permission information is available at http://www.nature.com/reprints

Publisher's note Springer Nature remains neutral with regard to jurisdictional claims in published maps and institutional affiliations.

\section{(c) (1)}

Open Access This article is licensed under a Creative Commons Attribution 4.0 International License, which permits use, sharing, adaptation, distribution and reproduction in any medium or format, as long as you give appropriate credit to the original author(s) and the source, provide a link to the Creative Commons license, and indicate if changes were made. The images or other third party material in this article are included in the article's Creative Commons license, unless indicated otherwise in a credit line to the material. If material is not included in the article's Creative Commons license and your intended use is not permitted by statutory regulation or exceeds the permitted use, you will need to obtain permission directly from the copyright holder. To view a copy of this license, visit http://creativecommons.org/ licenses/by/4.0/.

(C) The Author(s) 2021 